\title{
Improved Upper Bounds for Self-Avoiding Walks in $\mathbb{Z}^{d}$
}

\author{
André Pönitz and Peter Tittmann \\ Hochschule Mittweida \\ 09648 Mittweida \\ Technikumplatz 17 \\ Germany \\ e-mail: peter@htwm.de, poenitz@htwm.de
}

Submitted: June 7, 1999; Accepted: April 13, 2000

AMS Classification: 82B41, 05A15

\begin{abstract}
New upper bounds for the connective constant of self-avoiding walks in a hypercubic lattice are obtained by automatic generation of finite automata for counting walks with finite memory. The upper bound in dimension two is 2.679192495 .
\end{abstract}

\section{Introduction}

Counting self-avoiding walks in a hypercubic lattice $\mathbb{Z}^{d}$ is one of the hardest open problems in combinatorics. Walks of length up to 51 have been enumerated by Conway and Guttmann [3] with considerable computational effort. Obviously, the number $f^{(d)}(n)$ of self-avoiding walks of length $n$ in $d$ dimensions is at least $d^{n}$ by allowing only steps in each positive direction. On the other hand, a lower bound of $2 d(2 d-1)^{n-1}$ can be obtained by counting walks without immediate reversals. The connective constant

$\mu^{(d)}=\lim _{n \rightarrow \infty} \sqrt[n]{f^{(d)}(n)}$ is therefore bounded by the trivial bounds $d$ and 
$2 d$. Tighter rigorous bounds for $\mu^{(d)}$ are presented in the famous book by Madras and Slade [7]. The latest improvements in the field can be found in [8] achieving upper bounds of 2.6939 and 4.7476 in two and three dimensions, respectively, by an application of the Goulden-Jackson cluster method [6].

In this paper, we improve these bounds to 2.6792 and 4.7387 , respectively, without using the Principle of Inclusion and Exclusion employed by Goulden and Jackson. The main idea is as follows. Let $k, n$ be integers and $k$ even. Define $g^{(d, k)}(n)$ to be the number of walks of length $n$ in dimension $d$ that have at least one loop of length $k$ or less. Given a fixed $k$, we construct a finite automaton $A^{(d, k)}$ to generate the numbers $g^{(d, k)}(n)$.

Since a self-avoiding walk does not contain loops of any length, we can obtain an upper bound for the number $f^{(d)}(n)$ of self-avoiding walks by simply subtracting $g^{(d, k)}(n)$ from the total number of walks of length $n$-i.e. $(2 d)^{n}$.

The ordinary generating function $G^{(d, k)}(z)=\sum_{n} g^{(d, k)}(n) z^{n}$ can be derived by solving a system of equations created from the automaton's state transfers. The resulting function is always a rational function whose asymptotic behavior is determined by the root of smallest modulus of the denominator polynomial. This root can be obtained by iterating the automaton itself in order to avoid processing of huge matrices.

\section{The Automaton}

We demonstrate the construction of the automata $A^{(d, k)}$ in the special case $k=4$ and unspecified $d$. The state set of the automaton is defined as follows.

The initial state 0 corresponds to the unique walk of length zero. The final state $\omega$ represents walks that have a complete loop of length up to $k=4$ (i.e. 2 or 4 , since there must be an even number of steps in the loop). This state is absorbing (and the only absorbing state of the automaton for that matter) since a walk with a loop never loses this property. All other states represent classes of walks that exhibit a certain pattern in their last few steps. We construct them one by one, always trying to keep the automaton as small as possible. In order to extend the walk of length zero by one step, we can go in each of the $2 d$ possible directions. This could be handled by $2 d$ different states. However, all these different situations can be characterized the same way: We have a walk whose history can't contribute to a loop of length up to 4 . In this case this is simply be because there is no history at all, but later there will be other walks in state 1 as well. Therefore we create only one new 
state 1 to represent all those walks of any length $n$ whose first $n-1$ steps cannot contribute to a loop of length less or equal to 4 . Obviously, all the $2 d$ ways out of state 0 lead to state 1 . We will see later that this classification preserves all necessary details for our purpose.

Suppose, our walk is in state 1. There are three possibilities to continue: (1.1) The next step is an immediate return, thus closing a loop of length 2 and transfering us directly to state $\omega$. (1.2) We proceed in the same direction. Consider the previous step. In order to make this step part of a loop we have to walk back to its start somehow. In case of an immediate return we would close a loop of length 2 that does not use the previous step; in any other case we are not able to finish the loop with four steps or less. In any case, the previous step is not part of the first loop of length 2 or 4 for any continuation of our walk, so it is not really neccessary to preserve the information that the previous step was taken at all - except for its contribution of 1 for the total length of the walk of course. But this means we are effectively transfered back to state 1 again. (1.3) In the remaining $2 d-2$ cases the walk turns into another dimension. We represent this configuration by a new state 2 .

Suppose now, we have a walk in state 2. This time there are four ways to continue: (2.1) Immediate return transfering the walk to state $\omega$. (2.2) A step in the same direction as the previous step, transfering us back to state 1 for the same reasons as mentioned above under (1.2). (2.3) A step in the opposite direction to the second last step, transfering as to a new state 3 representing all those walks whose last three steps form a $U$-shape. (2.4) A step in any other of the remaining $2 d-3$ directions, transfering again to state 2 for similar reasons as above.

Last but not least, the possible transfers from state 3 are: (3.1) Immediate return closing a loop of length 2 or a step to the start of the third last step closing a loop of length 4 - both transfering to state $\omega$. (3.2) A step in the same direction as the previous step, transfering us back to state 1 again. (3.3) A step in any other of the remaining $2 d-3$ directions, all transfering to state 2.

The automaton can be translated directly into a system of equations for the generating function. Let $G_{i}^{(d, 4)}(z)$ be the ordinary generating function for the number of walks in state $i$ and $z$ a formal variable representing one 


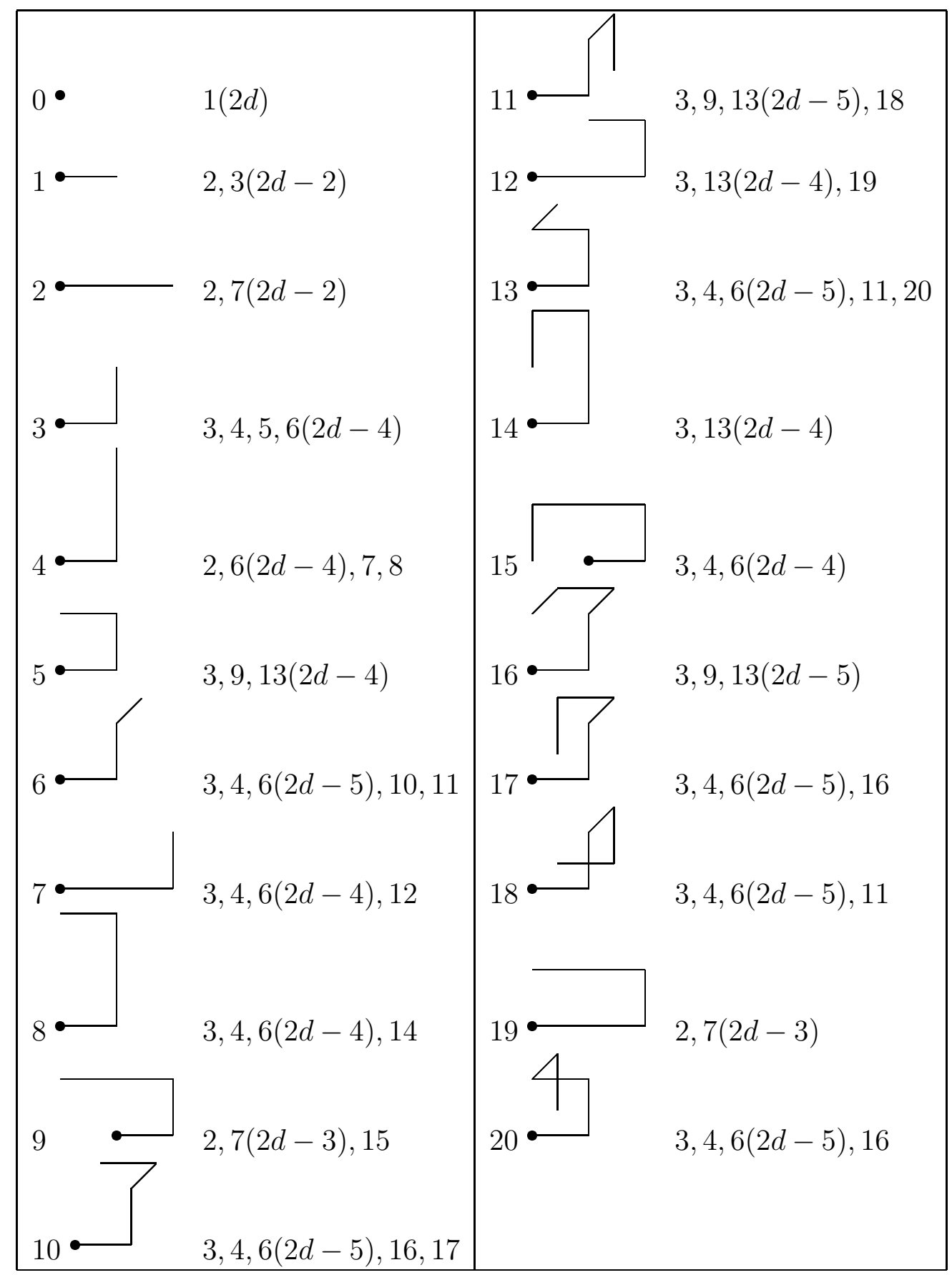

Table 1: States and transfers of the automaton $A^{(d, 6)}$. This incorporates already the simplifications by symmetry mentioned in the text. The commaseperated numbers are the state numbers of the successor states, a number followed by another number in parantheses indicates several directions leading to the same successor. Transfers to the final state $\omega$ are not shown. 


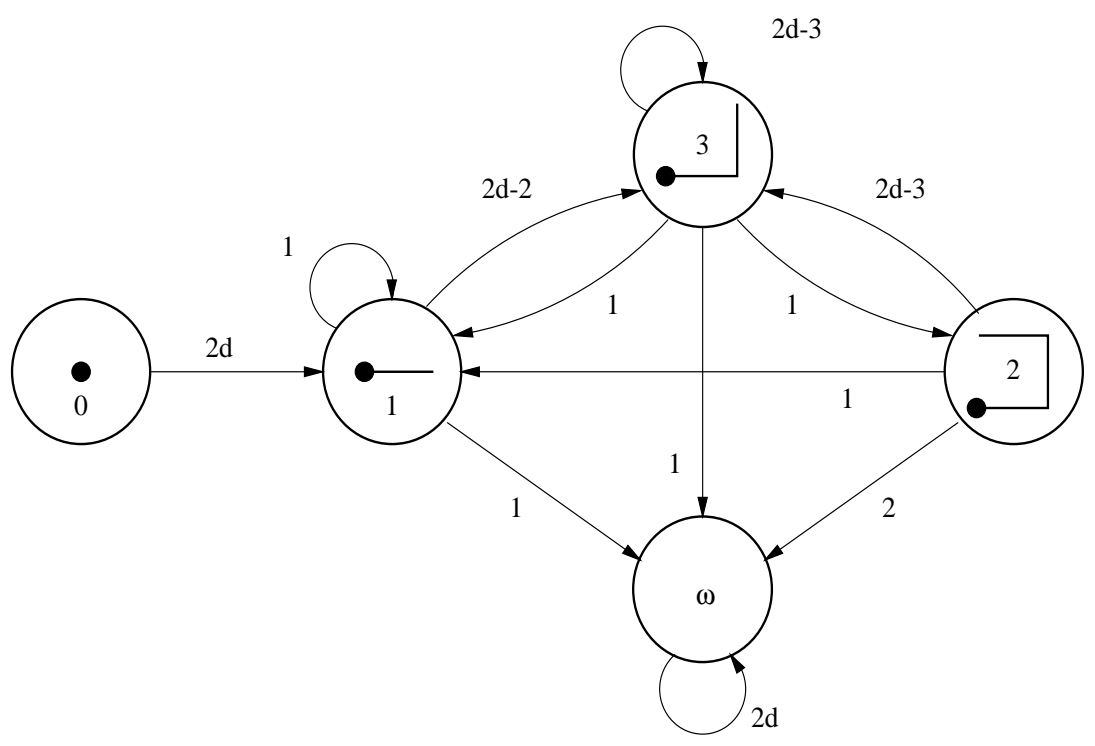

Figure 1: The automaton $A^{(d, 4)}$.

step of a walk. Then:

$$
\begin{aligned}
& G_{0}^{(d, 4)}(z)=1 \\
& G_{1}^{(d, 4)}(z)=z\left[2 d G_{0}^{(d, 4)}(z)+G_{1}^{(d, 4)}(z)+G_{2}^{(d, 4)}(z)+G_{3}^{(d, 4)}(z)\right] \\
& G_{2}^{(d, 4)}(z)=z\left[(2 d-2) G_{1}^{(d, 4)}(z)+(2 d-3)\left(G_{2}^{(d, 4)}(z)+G_{3}^{(d, 4)}(z)\right)\right] \\
& G_{3}^{(d, 4)}(z)=z\left[G_{2}^{(d, 4)}(z)\right] \\
& G_{\omega}^{(d, 4)}(z)=z\left[2 d G_{\omega}^{(d, 4)}(z)+G_{1}^{(d, 4)}(z)+G_{2}^{(d, 4)}(z)+G_{3}^{(d, 4)}(z)\right]
\end{aligned}
$$

From this we obtain easily

$$
F^{(d, 4)}(z)=\frac{1+2 z+2 z^{2}-z^{3}+2 z^{3} d}{1-2 z d+2 z-2 z^{2} d+2 z^{2}-z^{3}}
$$

The roots of the denominator polynomial $1-2 z d+2 z-2 z^{2} d+2 z^{2}-z^{3}$ can be found explicitly yielding some rather ugly explicit algebraic expression depending on $d$. In any case, numerical approximations of the roots for specific values of $d$ are found easily, and so are the inverses of the roots of smallest modulus giving us the entries of first line of table 2 . 
The manual construction of automata for walks with memory greater than eight is no longer feasable, since the number of states increases exponentially. Fortunately, automating the generation of states and transfer matrices is straightforward as the following algorithm shows:

1. Initalize a set of untreated states with state 0 as the only element, an empty set of treated states, and an empty set of transfers.

2. Choose any untreated state $s$, remove it from the set. This state represents a certain class of walks $w_{i}$ with individual lengths $l_{i}$ whose first $l_{i}-l$ steps cannot contribute to loop of length less or equal to the memory $k$ and whose last $l$ steps are identical. These last $l$ steps form a walk by their own that could be considered as a representative $r$ of the state. Because of the limited memory $k$, all of these walks will behave exactly the same in the future, and thus it is sufficient to identify the state with its representative and to keep track of the number of walks in the state instead of all the walks themselves.

3. Construct all possible successors of the state by iterating through all $2 d$ possible directions. In each iteration, augment $r$ by a single step in the corresponding direction, leading to an augmented walk $a$. If there is any possibility to form a loop of length less or equal to $k$ starting with $a$, then $a$ is the representative of a successor state $t$ of $s$. If $t$ is a state we have not seen so far, put it in the set of untreated states. In any case, keep track of the transfer from $s$ to $t$ in the set of transfers. If there is no possibility to form a loop of length less or equal to $k$ starting with $a$, then we start removing steps from the beginning of $a$ until we arrive at a walk $a^{\prime}$ that could be the first part of such a loop. Note that this procedure terminates since the length of $a$ is finite at the beginning, shrinks by 1 in each iteration, and the empty walk surely is extentable to a loop within $k$ steps. Now the walk $a^{\prime}$ is a representative of some state $t^{\prime}$ that is handled exactly like the state $t$ in the other case.

4. Put state $s$ into the set of treated states. If the set of untreated states is not empty, go to 2 , otherwise go to 5 .

5. We now have collected all necessary states in the set of treated states and all transfers in the set of transfers, thus the automaton is built. Note that the outer loop terminates since there is a (generous) upper 
bound of $(2 d)^{k}$ to the number of possible states and every iteration of the loop deals with one of them.

This base algorithm can be improved by combining sets of similar states. These improvements are not strictly necessary for the subsequent theoretical examinations. For practical computations, however, they are indispensable since they reduce the size of the automata thus saving valuable memory and increasing general performance.

One possibility is to exploit symmetry in the states. If one walk uses only the first $j<d$ out of $d$ possible dimensions, it is sufficient to consider the $2 j$ extensions staying completely in those $j$ dimension and a single other one going on step in positive direction in dimension $j+1$.

In our implementation we exploit this kind of symmetry by "normalizing" states. A normal state is a state representing walks that do not touch dimension $i$ until it has touched all dimensions $j<i$. States whose normalized representatives are the same could be collected since they exhibit equal behaviour in the future.

Another possibility is to trade off the size of the automaton against run time. To answer the question whether it is possible to close a loop in a given number of steps with the side condition that certain points have to be avoided is time-critical. In theory, the solution is Dijkstra's shortest path algorithm. Practically, this is too slow. By loosening the side conditions, the algorithm generates a few states too much, but the check could be done by computing the Manhattan norm of the difference of start and end point of the walk instead of running a full-blown shortest path algorithm.

It should be stressed at this point that even if the above mentioned improvements to the base algorithm do modify the constructed automata (both in state set and transfer matrix), the changes do not bias the following explanations.

\section{Upper bounds for the connective constant}

The dimension of the system of equations complicates the explicit construction of the generating function for walks with memory greater than eight. However, the determination of the asymptotic behavior of the number of walks of finite memory is much easier.

We can use the automaton directly for computing the number $f^{(d, k)}(n)$ of self-avoiding walks with memory $k$ and length $n$ by introducing a new state $s$ 
summing all states with the exception of $\omega$, placing a single label at time 0 in state 0 and iterating the automaton step by step. Every number $\mu^{(d, k)}(n):=$ $\sqrt[n]{f^{(d, k)}(n)}$ is an approximation for $\mu^{(d, k)}$ and thus an approximation for an upper bound to $\mu^{(d)}$.

The generating function $F^{(d, k)}(z)=\sum_{n} f^{(d, k)}(n) z^{n}$ is a rational function. Thus the desired information is obtained by considering the root of smallest modulus of the denominator polynomial. Using Cramer's rule to solve the system of equations, we find that the denominator polynomial is completely given by the determinant of the matrix $I-z A$ where $I$ denotes the identity matrix and $A$ is the state transfer matrix of the automaton. Consequently, the root of smallest modulus of the denominator polynomial of the generating function is given by the largest real eigenvalue ${ }^{1}$ of $A$.

This eigenvalue can be obtained by the power iteration method described e.g. in [2]. Translating this method into the language of the automata is easy: If we start the power iteration with the initial vector $(1,0, \ldots, 0)$ and identify state $i$ of the automaton with position $i+1$ in the iterated vector then the $n$th iterated vector is identical to the state of the automaton after the $n$th iteration.

A theorem by Hammersley and Morton cited in [7] guarantees the existence of the limit $\mu^{(d, k)}=\lim _{n \rightarrow \infty} \mu^{(d, k)}(n)$. Computing $\mu^{(d, k)}$ using this formula directly is awkward since convergence is slow and we don't even know anything about the rate of convergence. However, we can do better by using a slightly amended power iteration method. Observe that the graphs of transfers of any of the constructed automata has a single strongly connected component. Moreover, the state corresponding to $k / 2-1$ steps in the same direction is a recurrent state and there is a transfer from this state to itself. So, after $k / 2-1$ iterations of the automaton, the number of walks in this state is larger than zero, and the state serves as "pump" such that after at most $k-1$ iteration there is a non-zero number of walks in each of the states of the strongly connected component. Reducing the automaton to the states in the strongly connected component does not change the value of its largest eigenvalue; moreover, by combining $k-1$ iterations to a single transfer we obtain a strictly positive transfer matrix. This way a geometrical convergence of the power iteration method using this new transfer matrix is guaranteed.

\footnotetext{
${ }^{1}$ This approach is similar to the ideas presented by Alm [1], except that our matrices are not (yet) positive.
} 
THE ELECTRONiC JOURNAL of COMBinAtorics 7 (2000), \#R21

\begin{tabular}{|c|rrrr|}
\hline$k \backslash d$ & 2 & 3 & 4 & 5 \\
\hline 4 & 2.8312 & 4.8646 & 6.8917 & 8.9105 \\
6 & 2.7756 & 4.8075 & 6.8513 & 8.8816 \\
8 & 2.7445 & 4.7780 & 6.8303 & 8.8679 \\
10 & 2.7248 & 4.7599 & 6.8179 & 8.8602 \\
12 & 2.7113 & 4.7476 & 6.8097 & \\
14 & 2.7014 & 4.7387 & 6.8040 & \\
16 & 2.6939 & & & \\
18 & 2.6880 & & & \\
20 & 2.6832 & & & \\
22 & 2.6792 & & & \\
\hline
\end{tabular}

Table 2: Upper bounds for the connective constants $\mu^{(d, k)}$ for walks with finite memory. The values shown are true upper bounds.

\section{Acknowledgements}

We thank Tony Guttmann and the unknown referee who read the first version of this paper for their comments and suggestions.

\section{References}

[1] Alm, Sven Erik: Upper bounds for the connective constant of self-avoiding walks, Combinatorics, Probability and Computing, (1993) 2, 115-136

[2] Chatelin, Françoise: Eigenvalues of matrices, John Wiley \& Sons, Chichester, 1993

[3] Conway, A.R. and A.J. Guttmann: Square lattice self-avoiding walks and corrections to scaling, Physical Review Letters, 77(1996), 5284-5287

[4] Feller, W.: An introduction to probability theory and its applications, vol. I, Wiley, New York, 1968

[5] Fisher, M.E. and M.F. Sykes: Excluded-volume problem and the Ising model of ferromagnetism, Phys. Rev. 114, 45-58, 1959

[6] Goulden, I. and D.M. Jackson: Combinatorial enumeration, John Wiley, New York, 1983 
[7] Madras, Neal and Gordon Slade: The self-avoiding walk, Birkhäuser, Boston, 1993

[8] Noonan, John: New upper bounds for the connective constants of selfavoiding walks, Journal of Statistical Physics, p.871-888, Vol. 91, 1998

[9] Wall, F.T. and R.A. White: Macromolecular configurations simulated by random walks with limited order of non-self-intersections, J. Chem. Phys. 65, 808-812, 1976 